\title{
GAIA Level 1 Maternal Death
}

National Cancer Institute

\section{Source}

National Cancer Institute. GAIA Level 1 Maternal Death. NCI Thesaurus. Code C127997.

GAIA Level 1 Maternal Death is defined by three criteria: first, diagnosis of a pregnancy as established by any one of the following documented requirements: a) Ultrasound examination; b) Fetal heart tones; c) Positive serum or urine human chorionic gonadotropin pregnancy test; d) Delivery of a neonate or other products of conception (abortus, stillborn); second, the death of the mother while pregnant or within 42 days of the termination of a pregnancy, irrespective of the duration and the site of the pregnancy; third, documentation of the maternal cause of death as one of the following: a) Direct: abortive outcome, hypertensive disorder, obstetric hemorrhage, pregnancy related infection, other obstetric complications, unanticipated complications; b) Indirect: non obstetric complications; c) Death during pregnancy, childbirth, and the puerperium: other or coincidental. 